Meta

Journal des traducteurs

Translators' Journal

\title{
La traduction littérale : une statue aux pieds d'argile
}

\section{J. M. Babut}

Volume 32, numéro 1, mars 1987

La traduction biblique

Bible Translation

URI : https://id.erudit.org/iderudit/001935ar

DOI : https://doi.org/10.7202/001935ar

Aller au sommaire du numéro

Éditeur(s)

Les Presses de l'Université de Montréal

ISSN

0026-0452 (imprimé)

1492-1421 (numérique)

Découvrir la revue

Citer cet article

Babut, J. M. (1987). La traduction littérale : une statue aux pieds d'argile. Meta, 32(1), 26-29. https://doi.org/10.7202/001935ar d'utilisation que vous pouvez consulter en ligne.

https://apropos.erudit.org/fr/usagers/politique-dutilisation/ 


\section{LA TRADUCTION LITTÉRALE : UNE STATUE AUX PIEDS D'ARGILE}

J.M. BABUT

Société biblique française, Paris, France

La Bible n'est connue du grand public qu'à travers des traductions. Le lecteur francophone d'une version traditionnelle, surtout s'il utilise une édition d'étude largement annotée, verra son attention attirée par un nombre non négligeable de notes doublant la traduction adoptée de tel mot ou de telle expression par une autre traduction introduite comme «littérale».

\section{DES « NOTES DE LITTÉRALITÉ "}

Cette pratique est relativement originale. Les traducteurs de romans, de traités ou même d'ouvrages théologiques ne recourent en effet pour ainsi dire jamais à ce type de note. En revanche certaines éditions bibliques en sont assez prolixes. Nous avons ainsi comparé les traductions du livre de Ruth dans trois versions françaises qui ont pignon sur rue :

Bible de la Pléiade (BP, trad. Ed. Dhorme, 1959)

- Traduction OEcuménique de la Bible (TOB, 1975)

- Bible à la Colombe (BàC, Nouvelle version Segond révisée, 1978)

Sur 74 notes, BP en consacre 18 (soit $24 \%$ ) à donner une traduction littérale. TOB en propose 14 sur 45 (soit 32\%) et BàC 15 (voire 19) sur 51 (soit 29\% voire 37\%).

Dans l'ensemble des trois versions, 41 mots ou expressions du livre de Ruth font ainsi l'objet d'une "note de littéralité ", mais 8 cas seulement sont communs à deux ou aux trois versions. C'est dire que, dans la plupart des cas, une de ces versions propose une "note de littéralité " là où les autres ne le jugent pas utile. Ainsi en 1.16, BP traduit : "N'insiste pas... pour que je te quitte en retournant loin de toi... » et indique en note : " loin de toi ", littéralement 'de derrière toi', tandis que TOB rend ainsi la même phrase : "Ne me presse pas de t'abandonner, de retourner loin de toi » et BàC: "Ne me pousse pas à te quitter, à me détourner loin de tes pas ", l'une et l'autre de ces deux dernières versions considérant la traduction proposée comme se suffisant à elle-même.

Plus inattendu : le même verset (2.18) fait par exemple l'objet en BP et BàC de deux "notes de littéralité " portant sur des points différents (nous soulignons les mots sur lesquels portent ces dernières). BP : "Elle montra et lui donna ce qu'elle avait eu de reste après s'être rassasiée " et BàC : "elle sortit aussi le surplus de son repas et le lui donna ". Pour elle montra, BP précise : littéralement " elle fit sortir » de l'endroit où elle avait déposé sa petite récolte. De son côté, BàC juge nécessaire, semble-t-il, de justifier l'élégance et la brièveté de la traduction qu'elle propose malgré la complexité relative de l'expression hébraïque correspondante. Sa note précise en effet : litt. « ce qu'elle avait de reste après s'être rassasiée ». Pour la TOB, la traduction proposée (ce qui lui était resté une fois rassasiée, elle le sortit et le lui donna) n'appelle pas d'éclaircissement.

\section{DES CORRESPONDANCES CONVENTIONNELLES}

Ces constatations amènent à demander pourquoi le traducteur a jugé nécessaire d'ajouter un tel type de note ? L'exemple cité plus haut, emprunté à Ruth 2.18, permet

Meta, XXXII, 1, 1987 
de repérer les motifs non explicités de cette pratique. En traduisant "elle montra », BP estime nécessaire de corriger ou de préciser sa traduction, en notant que la traduction " littérale " exigerait qu'on écrive « elle fit sortir ». Or c'est précisément cette traduction qui est donnée dans le texte par TOB et BàC. On constate donc, entre les trois versions retenues, une sorte de consensus selon lequel à la racine hébraïque $y c ̧$ ' correspond obligatoirement le français sortir. Comme si la correspondance $y \zeta^{\prime} /$ sortir était exclusive et stéréotypée.

Le même phénomène se retrouve par exemple avec le mot descendance en 4.12 (BP : la descendance que te donnera Iahvé... TOB : la descendance que le Seigneur te donnera... BàC : la descendance que l'Éternel te donnera). BP et BàC estiment utile de noter que la traduction " littérale " serait la semence. Certes, pour éviter le non-sens, les traducteurs ont dû renoncer à semence au profit de descendance : mais, semble-t-il, non sans regret. Pour eux, la vraie traduction aurait dû être semence. Une fois encore, la règle non formulée établit une correspondance univoque et stéréotypée entre l'hébreu zèra 5 et le français semence.

Que penser de cette correspondance?

Robert distingue 4 acceptions de semence:

1. (a) : "organe ou fragment de végétal capable de produire un nouvel individu ". Dans certains cas, le terme équivaut à "graines que l'on sème".

1. (b) : À cette acception se rattache la signification figurée "Germe d'où naît quelque chose", comme dans l'expression "les semences d'une révolution".

2. "liquide séminal du mâle ", plus couramment appelé sperme (francisation du grec sperma).

Suivent deux acceptions techniques très particulières :

3. en joaillerie le terme désigne aussi, par relation métaphorique, un « ensemble de diamants ou de perles de très petite dimension".

4. Par relation métaphorique également, semence peut désigner enfin des clous " à tête plate et tige courte utilisés par les tapissiers".

De son côté l'hébreu zèra 5 a une gamme d'emplois passablement différents. Outre la semence, le terme peut désigner aussi les semailles. De l'acception "semen virile ", qui correspond d'ailleurs à un emploi possible mais rare du français, l'hébreu permet de passer, par une relation d'ordre métonymiquel à l'acception descendance et aux acceptions proches de tribu, race ou dynastie, ce qui ne correspond pas aux possibilités de semence.

Entre zèra 5 et semence, il n'a donc que deux acceptions communes. Pour toutes les autres acceptions de l'hébreu ou du français, les phénomènes métaphorique et métonymique ont fonctionné dans des directions différentes et, dans ce cas, les deux mots ont pris des significations sans correspondance mutuelle.

Dans ces conditions, pourquoi privilégier le rapport zèra5/semence? Considérerait-on qu'un mot a un sens "fondamental " autour duquel s'articulent les autres ? Mais lequel définir comme tel ? Le sens primitif doit-il être le sens " concret ", ou le sens "inanimé "? Mais sur quelle base prétendre que le sens premier est celui-là ? Privilégier le rapport zèra5/semence correspondrait-il à la distribution statistique ? -- Nullement ! Sur environ 230 occurrences de zèra5 dans l'Ancien Testament hébreu, seules 44 (soit 9\%) correspondent aux acceptions semence ou semailles, l'écrasante majorité des cas (161, soit 70\%) correspondant aux acceptions descendance, race ou dynastie.

Prétendre que semence est le sens littéral (premier ?) de l'hébreu zèra 5 paraît donc pour le moins arbitraire. On ne voit guère alors comment la traduction déclarée « litté- 
rale " peut aider le lecteur (qui n'est pas censé connaître l'hébreu biblique) à cerner avec plus de précision la signification originale. Tout au plus peut-on ainsi lui suggérer que l'hébreu et le français développent leurs métaphores respectives, d'ailleurs mortes à ce stade, dans des directions différentes. Mais cela n'est-il pas le propre de toutes les langues ? Tout traducteur, en effet, s'est rapidement aperçu qu'il était exceptionnel de pouvoir transposer telle quelle une métaphore d'une langue dans une autre et que la seule voie respectueuse de la signification consistait à chercher non pas l'identique mais l'équivalent.

\section{TOURNURES IDIOMATIQUES}

Dans une huitaine de cas, nous l'avons vu, une même tournure fait l'objet d'une note de littéralité de la part de deux ou des trois versions considérées. Fait frappant, il s'agit dans tous les cas d'expressions idiomatiques. Encore faut-il observer que les solutions adoptées diffèrent parfois d'une version à l'autre. Nous nous arrêterons sur deux exemples.

En 4.4, lorsque Booz, siégeant "à la porte de la ville ", c'est-à-dire au conseil des anciens, négocie le rachat de Ruth et de sa propriété avec celui qui dispose en priorité du droit de rachat, il lui dit, selon BP : «...j'ai dit que je t'en informerai »; selon TOB : « j'ai dit que je te mettrai au courant " et, selon BàC : «j'ai pensé t'en informer ». Les trois versions ont jugé utile de donner en note la traduction " littérale " de l'expression idiomatique de l'hébreu rendue par informer ou mettre au courant, à savoir "découvrir l'oreille ". Ce type de note ajoute certes une touche de pittoresque, mais n'éclaire en rien la signification de l'expression hébraïque. Tout est déjà dit par le verbe informer ou par la tournure idiomatique mettre au courant. Si l'on estime intéressant d'avoir un décalque français de l'expression hébraïque, il paraît utile alors de préciser que "informer quelqu'un » ou "mettre quelqu'un au courant " est l'équivalent français de "découvrir l'oreille de quelqu'un ». Sous ce rapport, les notes de BP, TOB et BàC sont insuffisantes.

Le second exemple est emprunté à 3.9. À l'inverse de 2.18, BàC est ici la plus « littérale " des trois versions : "étends ton aile sur ta servante ". La difficulté de l'expression est double. Il y a d'abord l'usage métaphorique d'aile pour désigner le pan (du manteau). Devant la phrase "étends ton aile sur ta servante", le lecteur francophone non averti percevra bien qu'il doit comprendre ici aile au sens figuré. Mais, cherchant la signification de cette métaphore, il la comprendra sans doute comme une demande de protection, à l'image de la poule qui abrite ses poussins sous son aile. À aucun moment il n'aura l'idée que Ruth fait allusion au pan du manteau de Booz, car le français n'a pas développé les valeurs métaphoriques du mot aile dans le même sens que l'hébreu pour le mot kanaf.

La seconde difficulté est d'ordre culturel. Le geste décrit ici symbolise, de la part d'un homme, la volonté d'épouser la femme qu'il a ainsi abritée sous son manteau. Ce symbole, étranger à la culture des lecteurs francophones, devra être explicité. BP et BàC l'ont fait dans une note. Mais, du point de vue de la traduction proprement dite, la solution la plus satisfaisante est celle proposée par la TOB : «Épouse-moi ». À ceci près cependant que TOB rend de façon abstraite ce que l'hébreu exprimait de façon idiomatique $^{2}$.

\section{LES PRÉSUPPOSÉS DE LA TRADUCTION "LITTÉRALE »}

Pour terminer, il faut s'interroger sur les présupposés de cette pratique si répandue. À ma connaissance, ils n'ont jamais fait l'objet d'une analyse méthodique. On doit donc s'avancer ici avec prudence.

Un des premiers présupposés de ce type de traduction est, semble-t-il, le caractère sacré attribué au texte biblique original. Dans la mesure où chaque lettre de ce texte est 
considérée comme inspirée, le respect que, dans cette perspective, on doit au texte biblique se reporte sur les mots mêmes de ce texte. On en arrive très vite à conférer à ces mots pris en eux-mêmes un poids déterminant, que la traduction " littérale " cherche à mettre en valeur.

La même conséquence résulte aussi, me semble-t-il, du désir de faire apparaître dans la traduction les rapports d'intertextualité, comme on dit aujourd'hui : tel mot clé d'un passage évoque, pour le lecteur, un autre passage où figure le même mot. Il met ainsi les deux textes en relation et transfêre dans le premier les significations véhiculées par le second. Dans une telle perspective, il importe donc qu'un même mot de l'hébreu ou du grec soit, si possible, toujours rendu par le même mot français et réciproquement, de façon à permettre les rapprochements. Cette préoccupation est sans doute à l'origine de la convention que nous avons relevée plus haut selon laquelle $y c ̧$ ' doit toujours être sortir, zèra 5 : semence, etc.

Ces présupposés aboutissent, comme on le voit, à conférer aux mots pris en euxmêmes un poids de signification considérable. Or l'analyse sémantique de la portée d'un mot dans une phrase montre qu'il s'agit là d'une surévaluation certaine. En lui-même un mot n'est rien, précisément parce qu'il est susceptible de plusieurs voire de trop nombreuses acceptions. Seule la phrase - ou le paragraphe - dans laquelle il s'articule permet de sélectionner l'acception unique que prend tel mot dans un contexte donné (à la rigueur deux acceptions, s'il y a ambiguïté ou jeu de mots intentionnel). Le mot en luimême reste insuffisant à déterminer la signification. Celle-ci résulte aussi du contexte dans lequel ce mot est articulé. Sauf, bien entendu, si le terme se charge de la même acception en deux passages distincts, il ne sert à rien de vouloir retrouver en français les récurrences des mots hébreux ou grecs.

C'est pourquoi la traduction «littérale », construite ainsi sur une base linguistique erronée, est aussi fragile que la statue aux pieds d'argile.

Notes

1. Avec M. Le Guern (1973) : Sémantique de la métaphore et de la métonymie, Paris, p. 28, nous refusons de voir une différence de nature entre synecdoque et métonymie. Dans ce qui suit, nous employons métonymie au sens large, incluant synecdoque.

2. La traduction de la Bible en français courant (BFC 1982) nous paraît insuffisante : "Veuille me prendre sous ta protection." 